\title{
e-Phaïstos
}

e-Phaïstos

Revue d'histoire des techniques / Journal of the history

of technology

I-2 | 2012

Les sources de l'histoire des techniques

\section{Éric Rieth, Le maître-gabarit, la tablette et le trébuchet. Essai sur la conception non-graphique des carènes du Moyen Âge au XX ${ }^{\mathrm{e}}$ siècle}

\section{Benjamin Ravier-Mazzocco}

\section{(2) OpenEdition}

\section{Journals}

Édition électronique

URL : http://journals.openedition.org/ephaistos/443

DOI : 10.4000/ephaistos.443

ISSN : 2552-0741

Éditeur

IHMC - Institut d'histoire moderne et contemporaine (UMR 8066)

\section{Édition imprimée}

Date de publication : 1 décembre 2012

Pagination : 87-92

ISSN : 2262-7340

\section{Référence électronique}

Benjamin Ravier-Mazzocco, «Éric Rieth, Le maître-gabarit, la tablette et le trébuchet. Essai sur la

conception non-graphique des carènes du Moyen Âge au XXe siècle ", e-Phaïstos [En ligne], I-2 | 2012 mis en ligne le 12 février 2016, consulté le 24 septembre 2020. URL : http://journals.openedition.org/ ephaistos/443 ; DOI : https://doi.org/10.4000/ephaistos.443 


\title{
Éric Rieth, Le maître-gabarit, la tablette et le trébu- chet. Essai sur la conception non-graphique des ca- rènes du Moyen Âge au XX' siècle, (Paris, CTHS, coll. Histoire des sciences, 1996, 230 pages)
}

\author{
Benjamin Ravier-Mazzocco \\ doctorant au Centre d'Histoire des \\ Techniques (CH2ST/EA 127), \\ Université Paris 1 Panthéon-Sorbonne
}

La préface de Michel Mollat du Jourdin résume assez bien les principales caractéristiques de ce livre de référence sur l'histoire de la conception et construction des carènes, c'est-à-dire la partie immergée de la coque d'un bateau : une information riche, issue de traités, de résultats de fouilles sous-marines et d'enquêtes ethnologiques, permettent à l'auteur de mettre en avant un exemple passionnant d'un type de savoir et de transmission technique, que l'on pourrait qualifier, à la suite d'Anne-Françoise Garçon, de « régime pratique de la pensée opératoire ${ }^{1}$. L'apport majeur de ce travail est donc non seulement de montrer que la conception d'un objet, c'est-à-dire la partie la plus intellectuelle du processus de création technique, peut parfaitement s'appuyer sur un savoir non écrit, mais aussi d'en déployer les différentes modalités.

Fondé sur l'observation d'un maître, et le plus souvent d'un parent, l'apprentissage de l'usage du maîtregabarit, de la tablette et du trébuchet, outils transmis de génération en génération, est un type de transmission du savoir technique qui se situe à l'opposé d'un autre savoir de conception des carènes, de type technologique, fondé sur l'écrit et le calcul, et localisée hors des chantiers. Développé à partir de la fin du XVII e siè- cle, il reste longtemps cantonné à la construction navale militaire, la méthode traditionnelle perdurant dans la construction navale civile. Pourtant, c'est dans ces traités « technologiques » qu'Éric Rieth va d'abord chercher l'explication du fonctionnement de cette technique de conception. Le sommaire peut d'ailleurs sembler assez déroutant de ce point de vue, faisant succéder à une longue introduction sur la construction des carènes et son histoire avant le Moyen Âge, une analyse issue de traités des $\mathrm{XVII}^{\mathrm{e}}$ et $\mathrm{XVIII}^{\mathrm{e}}$ siècles, avant de revenir à des témoignages plus anciens, et de finir avec des enquêtes ethnographiques de la période contemporaine. Ces allers-retours se justifient pourtant, car ils permettent seuls au lecteur de comprendre l'aspect technique, indispensable à la compréhension de l'ouvrage dans sa totalité.

Le premier chapitre en effet, reprenant les résultats de sept fouilles archéologiques ${ }^{2}$ dans le bassin méditerranéen, permet de contextualiser la recherche en rappelant le passage, dans la méditerranée du VII ${ }^{\mathrm{e}}$ siècle, d'une construction de type "bordé premier » à une construction de type "membrure première ». Dans le premier cas, le navire est construit en posant les planches extérieures (le bordage), sur la quille, poutre 
centrale définissant le fond du bateau. Ensuite, le constructeur renforce ce bordage par des poutres intérieures transversales épousant la forme prise par le bordage. On appelle ces poutres des membrures. Dans le second cas, plus récent, les membrures sont construites d'abord, avant d'y fixer le bordage. C'est dans ce type de construction que se développe la méthode du maîtregabarit, de la tablette et du trébuchet.

Afin de bien comprendre l'enjeu de cette méthode, il faut d'abord bien saisir ce qu'elle permet de faire. Le nœud de la construction d'un bateau de type « membrure première » est la conception à l'avance, en fonction des caractéristiques du bateau qu'on souhaite construire, de la forme des couples. Un couple désigne l'ensemble des éléments caractérisant une membrure : la partie inférieure (varangue ou madier), la partie intermédiaire (genou), et la partie supérieure (allonge). Cette conception à l'avance des couples permet de gabarier les différents éléments et donc de distinguer dans le chantier naval, l'espace de la construction des éléments de l'espace de l'assemblage.

Ainsi, après avoir détaillé toutes les définitions de base dans le chapitre 2, Éric Rieth débute une nouvelle partie, fondée sur une série de traités théoriques de la période moderne, notamment ceux de Duhamel du Monceau, de La Madeleine et de Georges Juan, qui permettent aux lecteurs de comprendre la méthode de conception et de construction utilisant le maître-gabarit, la tablette et le trébuchet. Tentons ici, avec l'auteur, d'en synthétiser le principe.

Dans le mode de pensée qui préside à l'usage du maître-gabarit, de la tablette et du trébuchet pour la construction navale, tous les couples d'un bateau se définissent par rapport au couple le plus large (où le bateau sera le plus large au fond), qu'on appelle aussi le " maître-couple », divisé en deux « maîtresses-sections ». En effet, une fois défini ce maître-couple, il ne s'agit plus que de jouer sur trois dimensions pour concevoir les autres couples.

Il y a d'abord l'acculement, désignant la partie inférieure de la varangue, qui repose sur la quille. Cette partie inférieure, presque plate sur le maître-couple, augmente avec l'éloignement du couple au maître couple vers l'avant, ou, plus encore, vers l'arrière (voir fig. 1, en bas, l'acculement correspond à l'espace a-o sur chaque varangue).

Il y a ensuite la réduction du plat. Le fond du bateau voit en effet sa largeur rétrécir vers l'avant et vers l'arrière. Cela se traduit par une réduction du fond intérieur (plat) des varangues avec l'éloignement du couple au maître-couple (voir fig. 1, en bas, le plat est la ligne v-v sur chaque varangue).

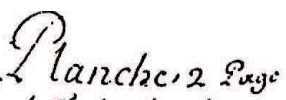
Uui repiceronle qua le Jiebuchet le quscmics; les

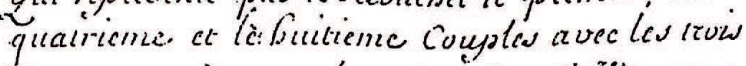
Haranguar de ar mémes couples. fig re 2 . page

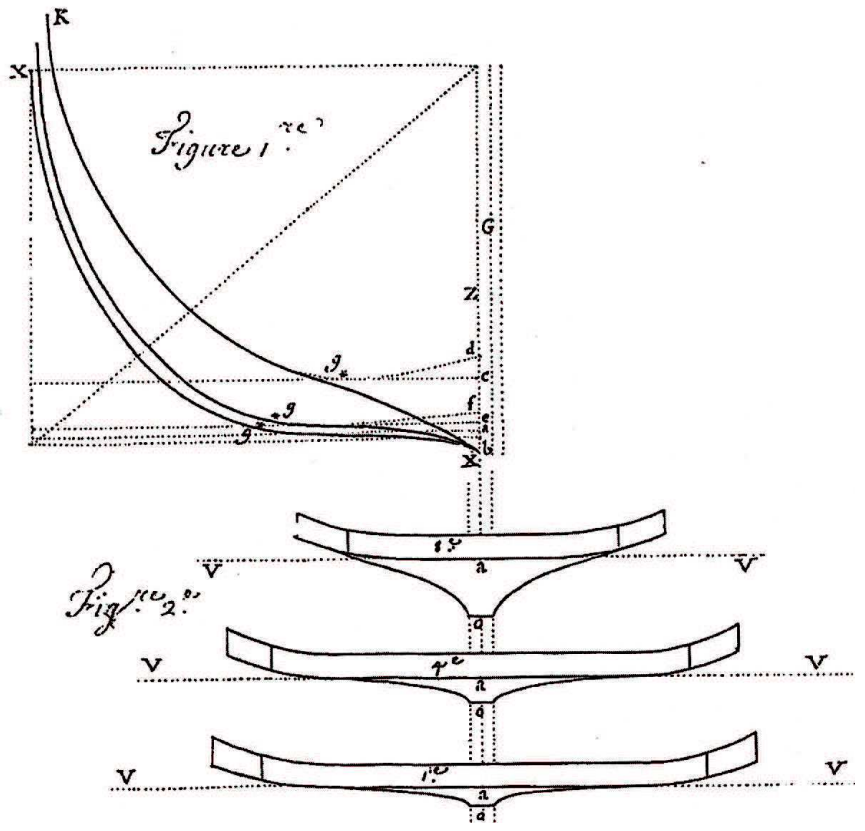

Fig. 1 : "Tracé du contour des membrures 1 , 4 et 8 selon La Madeleine » (LA MADELEINE (de), Tablettes de Marine, planche 2, Paris, Bibliothèque du musée de la Marine, ms. R711, vers 1712 ; cité dans le chapitre 4, "Le maîtregabarit, la tablette, le trébuchet », p. 7o) 
Il y a enfin l'ouverture. Afin de ne pas perdre en largeur sur les ponts du bateau ce qu'on perd en largeur au niveau du plat de la varangue, il est judicieux en effet de modifier l'angle d'ouverture du couple, c'est-à-dire de modifier la forme des allonges pour permettre à la largeur « en haut » d'être plus grande que la largeur « en bas » (voir fig. 1, en haut, le trébuchement permet une largeur presque similaire pour la varangue XK $\left(8^{\mathrm{e}} \mathrm{mem}-\right.$ brure) que pour la varangue XX ( $1^{\text {ère }}$ membrure)).

L'objectif, pour un constructeur naval, est alors de pouvoir gabarier la majeure partie des couples, en faisant varier, à partir de la maîtresse-section, la longueur du plat de la varangue, la hauteur de l'acculement et l'ouverture des couples. Avant d'arriver à la méthode du maître-gabarit, de la tablette et du trébuchet, il existe plusieurs stades, mais laissons d'abord Éric Rieth résumer cette méthode aboutie :

"Tout commence par une définition des dimensions sensibles de la coque selon un système de proportions simples faisant appel, en règle générale, à une dimension de référence (longueur de quille ou longueur de tête en tête notamment) variable selon la nature des bâtiments et les époques considérées. Intervient ensuite la détermination de la figure de la maîtressesection et son tracé en grandeur d'exécution sur le sol du chantier ou sur toute autre surface plane. À partir de ce tracé est confectionné le maître-gabarit, premier « instrument » de conception du constructeur, complété par les deux autres «instruments » que sont la tablette et le trébuchet.

[...] Pour la diminution de la longueur du plat de la varangue, le maître-gabarit est glissé latéralement selon un axe horizontal correspondant à celui de la ligne du plat. Pour l'augmentation de l'acculement, le maître-gabarit est déplacé verticalement au-dessus de la tablette d'acculement. Pour le trébuchement, le maître-gabarit est basculé latéralement à partir d'un point situé sensiblement au niveau du pied du genou. [...] Chaque « instrument » de conception porte une série de marques dont les intervalles évoluent progressivement. Ces marques correspondent aux différentes valeurs de la réduction de la longueur du plat, de l'augmentation de l'acculement et du trébuchement. La détermination de ces valeurs est effectuée à partir d'un diagramme à l'échelle 1 qui repose sur une construction géométrique élémentaire, dont l'une des plus anciennes est celle dite de la demi-lune. Les membrures localisées entre les sections de balancement [membrures placées à une distance stratégique du maître couple, au-delà desquelles la forme des membrures ne peut plus se définir par les trois opérations simples de modification des maîtresses-sections] et les deux pièces d'extrémité de la coque (étrave et étambot) sont définies, quant à elles, au cours de la construction, à partir des lisses [longues planches fines rejoignant l'avant et l'arrière du bateau en reliant l'extérieur de toutes les membrures déjà posées]. »3

Mais cette seconde partie va plus loin que la simple description de la méthode aboutie, « canonique ». Les théoriciens de la période moderne offrent en effet l'avantage de décrire des méthodes « antérieures », en réalité parallèles, de conception et de construction, qui permettent à Éric Rieth de décrire les différentes façons de définir les dimensions d'un navire selon son type, puis de construire un maître-gabarit, et de l'utiliser pour définir la forme des différents couples. Il décrit alors quatre stades d'évolution de cette méthode, la première ne se fonde que sur l'utilisation du maître-gabarit et de lisses dressées entre l'étrave, l'étambot et les maîtressessections permettant ainsi, directement dans l'espace, de définir les formes des autres membrures intermédiaires. L'histoire est ensuite celle de la conquête du tracé à l'avance des membrures intermédiaires, d'abord par l'ajout des couples de balancement, puis le contrôle, par les instruments, de la réduction de la largeur et l'augmentation de l'acculement, et enfin par le contrôle du trébuchement à l'avance. Éric Rieth rend ainsi compte d'une évolution du savoir de conception lié à ces objets, tordant le cou à une vision routinière de la transmission traditionnelle des techniques, il montre que l'innovation est aussi présente dans le régime pratique de pensée opératoire. En revanche, il s'attarde ici assez peu sur la façon dont les auteurs de la période moderne considéraient ces méthodes, traitées d'archaïques, et cherchaient à les remplacer par une conception entièrement réalisée hors du chantier, sur des plans à échelle réduite, par des ingénieurs spécialisés ${ }^{4}$. Il développe plutôt ces remarques sur le passage d'une conception non-graphique des carènes (celle du maître-gabarit) à une conception graphique (celle des plans) dans sa conclu- 
sion. C'est que le cœur du livre, et des parties suivantes, est justement occupé par la tradition désormais qualifiable de classique, du maitre-gabarit, de la tablette et du trébuchet : son objet n'est pas dans le passage d'un mode de pensée à l'autre, mais bien dans la caractérisation de l'évolution d'un mode de pensée traditionnel.

La troisième partie, organisée de manière régressive depuis les sources du XVII ${ }^{\mathrm{e}}$ siècle vers des sources les plus anciennes, vise à définir les origines de cette méthode de conception des carènes. Pour cela, Éric Rieth utilise d'abord différents manuscrits du $\mathrm{XV}^{\mathrm{e}}$ siècle vénitien au XVII ${ }^{\mathrm{e}}$ siècle espagnol. L'auteur se plaît ici à présenter les différentes variantes dans la définition des proportions générales en fonction des espaces et des auteurs. Surtout, tout en soulignant régulièrement l'extrême parenté de la pensée conceptrice à l'œuvre tant à Venise qu'en Espagne, il met en lumière les différentes méthodes pour déterminer, à l'aide de diagrammes de différentes sortes, dont la demi-lune est le plus ancien, les différentes valeurs d'augmentation de l'acculement et de réduction de la largeur du plat. Le chapitre dix clôt cette troisième partie sur une épave catalane, le Culip VI, dont les marques sur les madiers (varangues) permettent de faire remonter l'existence de l'usage d'un maître-gabarit, et d'une tablette au début du XIVe siè$\mathrm{cle}^{5}$, et sans doute même légèrement avant. De plus, cette épave témoigne du fait que cette technique n'était pas propre à l'arsenal de Venise, mais sans doute à une plus large part de la Méditerranée occidentale.

La quatrième et dernière partie, que le lecteur lit avec d'autant plus d'aisance qu'il est désormais familiarisé avec la technique du maître-gabarit, de la tablette et du trébuchet, reprend les témoignages d'un usage de ces outils aux $\mathrm{XIX}^{\mathrm{e}}$ et $\mathrm{XX}^{\mathrm{e}}$ siècles dans différents espaces : Marseille et le gabarit de Saint-Joseph, le lac Léman et ses barques, la Grèce et le monochnaro (un seul gabarit), le Brésil de Valença et du Nord de l'État de Bahia, et enfin Terre-Neuve de nos jours. Avec ce panorama, le lecteur comprend la longévité de la méthode du maître-gabarit, la diversité des variantes possibles, et voit le fait que certains stades d'utilisation de ces outils coexistent dans une même zone géographique. L'au- teur, quant à lui, peut développer ses vues plus générales sur le mode de transmission de cette technique et son évolution sur le long terme. Mettant en regard des témoignages oraux contemporains et des études ethnographiques, Éric Rieth nous montre en effet comment peut évoluer dans nos sociétés modernes un savoir ancien, issu d'un mode de pensée opératoire qui n'est plus celui qui domine le monde technique contemporain. D'abord, on apprend que l'usage d'une telle technique est resté vivace, malgré la fragilité d'un savoir transmis depuis l'enfance (10-12 ans), par « observation, imitation des gestes, contact progressif avec les matériaux, participation à des travaux d'abord élémentaires, puis de plus en plus compliqués, connaissance des outils, des « instruments » de conception, reconnaissance des " signes » inscrits sur ces « instruments », " mémorisation $» . . . »^{6}$, ce à quoi il faudrait ajouter une transmission, au sein de la cellule familiale des gabarits, qui forment, semble-t-il, le patrimoine le plus précieux des constructeurs de navires. Un patrimoine d'autant plus jalousement gardé que le maître-gabarit a une « dimension fondamentalement personnelle » ${ }^{7}$, qui signe là encore, le mode oral de pensée opératoire qui a perduré jusqu'à nos jours.

L'ensemble du livre est par ailleurs émaillé de remarques qui permettent de mieux comprendre en quoi réside le savoir-faire. Ainsi, en parlant de méthodes anciennes décrites par Lavanha, Éric Rieth explique comment « les « instruments » de conception se transforment alors en « instruments » de construction. Il s'établit de la sorte une relation logique, directe, entre le temps de la conception - celui de la réalisation du gabarit et de la tablette - et le temps de la construction, celui de l'utilisation du gabarit et de la tablette. $»^{8} \mathrm{Il}$ donne ici à voir ce qui est sans doute une des caractéristiques majeures du régime pratique de pensée opératoire. Plus intéressant encore est sans doute le passage sur le savoir de l'œil, chez les constructeurs de Terre-Neuve. Ainsi, l'auteur montre comment peut s'effectuer la création de certaines formes sans outils, grâce à la simple expérience accumulée du constructeur, qui se concentre dans un regard habitué aux formes: 
« La troisième méthode de conception en usage à Winterton consiste à recourir à des « gabarits intellectuels ", mental templates écrit D. A. Taylor ${ }^{9}$ ». Cette méthode consiste à sélectionner les parties des arbres ayant les formes les plus adaptées à un bateau dont les mesures ont été prédéfinies ; un choix qui respecte des normes implicites : « ce qui est correct, ce qui est bon, ce qui est juste, ce qui est harmonieux ${ }^{10}$. Les remarques sur le secret sont aussi très régulières et mettent souvent en avant, non sans raison, le caractère économique de la volonté de garder secret non seulement un savoir-faire, mais les outils qui permettent de l'exercer. Cependant, en s'attardant sur ce point, peut-être Éric Rieth ne voit-il pas (il faut dire que les indices sont ici fugaces) le rôle du secret comme suscitation du désir d'apprendre, notamment perceptible dans l'importance que revêt dans tous les exemples utilisés, le maître-gabarit, objet mystérieux et très personnel, nous l'avons vu, transmis d'une génération à l'autre. Il faut dire que c'est cet instrument qui cristallise, qui focalise tout le savoir-faire d'un constructeur de bateau, et qui en détermine sa valeur :

\begin{abstract}
«Son apprentissage achevé, un jour arrive où le jeune constructeur, qui est aussi pêcheur, c'est-à-dire utilisateur d'une embarcation, hérite de son père, de son frère ou de son oncle, un certain nombre de gabarits. Au début, il travaille en utilisant ses « instruments » de conception selon la méthode acquise au cours de son apprentissage. Puis, au fur et à mesure que son expérience de constructeur, mais également de pêcheur, augmente, il modifie, par approches successives et prudentes, sa manière de « penser » une embarcation [...]. La maîtrise de la méthode de conception héritée du père ou de l'oncle offre donc au constructeur expérimenté la possibilité de personnaliser sa façon de travailler en modifiant ses « instruments » [...]. Au terme de son activité professionnelle, ce sont ces « nouveaux » gabarits qu'il transmettra à son tour à l'un de ses fils ou de ses neveux ${ }^{11}$
\end{abstract}

Dans ce passage, Éric Rieth démontre aussi très bien que ces savoir-faire traditionnels ne sont pas bloqués, malgré ce qu'en pensent certains, à l'instar de Duhamel du Monceau, qui critique, comme c'était alors souvent le cas, la « routine » des artisans. L'analyse d'Éric Rieth est d'ailleurs intéressante à plus d'un titre, car elle lie les innovations possibles, non seulement à l'expérience et au savoir-faire acquis dans la construction des bateaux, mais aussi dans leur utilisation en navigation. Notons que cette illustration d'un savoir-faire local, et localement situé se retrouve aujourd'hui au cœur de certaines relocalisations, comme celle des skis « Rossignol », délocalisés en Asie, puis relocalisés en Haute-Savoie, proches des clients et donc des usagers.

Enfin, il convient d'insister ici sur les judicieuses remarques qu'Éric Rieth fait à propos de la fragilité de tels savoirs et savoir-faire, transmis par une tradition d'imitation. En effet, comme il le souligne, un arrêt prolongé de l'activité de construction peut empêcher la transmission de ce savoir de se faire correctement. Paradoxalement, le développement de l'école obligatoire et l'industrialisation des pièces détachées freinent aussi cette transmission. Si ces causes restent des hypothèses, la conséquence, elle, est bien visible dans le cas des constructeurs de Terre-Neuve qui, en l'espace de deux ou trois générations, ne savent plus utiliser les gabarits que pour la maîtresse-section, ne comprenant pas l'ensemble des marques disposées sur l'outil. Cela nous rappelle que des pans entiers de savoirs humains, de notre patrimoine commun, peuvent disparaître très rapidement.

Ce livre permet ainsi de retracer l'apparition, vers le XIV ${ }^{\mathrm{e}}$ siècle d'une technique de conception/ construction des carènes, sans plan ni support graphique (mis à part quelques proportions générales). Cette technique utilise trois instruments, qui utilisés ensemble permettent de tracer à l'avance les différentes membrures d'une coque de bateau. Plus encore, l'ouvrage d'Éric Rieth met en lumière comment, pendant les six siècles de son existence, cette technique évolue et se transmet par le geste, la parole et l'outil.

Un tel ouvrage, pour conclure, devrait être une référence non seulement pour les étudiants intéressés par l'histoire de la construction navale, mais aussi pour tout historien des techniques travaillant sur des techniques traditionnelles. Il propose en effet une méthode globale, qui utilise les sources écrites de l'historien, les résultats 
des fouilles archéologiques et les enquêtes ethnologiques orales, pour caractériser l'évolution d'une technique ancienne, transmise oralement, et donc difficile à saisir. Éric Rieth parvient ici à montrer ce à quoi il faut faire attention dans ce genre d'enquête, et notamment comment les outils, les instruments, qui servent de fil rouge aux recherches sur les différents types de sources, permettent de faire remonter à la surface un faisceau d'indices permettant de circonscrire les points importants des évolutions de techniques anciennes, et parfois oubliées.

${ }^{1}$ GARCON Anne-Françoise, " Ces techniques dont l'homme fait récit... De Verne à Fantomas, approches anthropo-historiques » dans CHOUTEAU Marianne et NGUYEN Céline (dir.), Mises en récits de la technique. Regards croisés, Lyon, éd. Archives contemporaines, 2011. Voici ce qu'elle dit du régime pratique de la pensée opératoire : « le fondement culturel du régime de la pratique est l'oralité. L'artisan développe autour et avec les gestes, instruments, procédés, qu'il met au point, utilise et transmet, une carte mentale dont l'objectif est à la fois de permettre le déroulement de l'action, de l'intégrer dans la cosmologie de son groupe humain ; et d'en favoriser la mémorisation et la transmission. 》

${ }^{2}$ Fouille des navires suivants, dans l'ordre d'analyse : Madrague de Giens (France, 70 av. J.-C.), Yassi Ada II (Turquie, IV ${ }^{\mathrm{e}}$ siècle), Kyrénia (IV ${ }^{\mathrm{e}}$ siècle av. J.-C.), la Bourse (France, II-III ${ }^{\mathrm{e}}$ siècle), Yassi Ada I (Turquie, VII ${ }^{\mathrm{e}}$ siècle), Saint-Gervais II (France, VII ${ }^{\mathrm{e}}$ siècle), Serçe Liman (Turquie, $\mathrm{XI}^{\mathrm{e}}$ siècle).

${ }^{3}$ Conclusion, p. 200-201.

${ }^{4}$ Cette dernière remarque est surtout vraie pour Duhamel du Monceau, les autres auteurs se rapprochant davantage d'une démarche proche de celle de la réduction en art. Voir sur ce sujet VERIN Hélène et DUBOURG-GLATIGNY Pascal, Réduire en art. La technologie de la Renaissance aux Lumières, Paris, éd. de la Maison des sciences de l'homme, 2008.

${ }^{5}$ L'absence des allonges (non conservées) sur l'épave ne permet pas de vérifier l'emploi d'un trébuchet.

${ }^{6}$ Conclusion, p. 207.

${ }^{7}$ Conclusion, p.206. Selon Éric Rieth, Duhamel du Monceau avait bien saisi cette dimension.

${ }^{8}$ Chapitre 8, « De l'usage du maître-gabarit et de la tablette selon J. B. Lavanha », p.131.

${ }^{9}$ Éric Rieth cite ici TAYLOR D. A., Boat building in Winterton Trinity Bay, Newfoundland, Ottawa, Centre canadien d'études sur la culture traditionnelle, dossier $n^{\circ} 41,1982$.
${ }^{10}$ TAYLOR D. A., 1982, op. cit. note 9, cité dans le chapitre 13, « Le témoignage des hommes de Terre-Neuve », p. 195.

${ }^{11}$ Chapitre 13, «Le témoignage des hommes de Terre-Neuve », p. 198. 